\title{
Hormonal replacement therapy in women with a history of internal genital organ malignancy
}

\author{
Maria Brzozowska, Andrzej Lewinski \\ Department of Endocrinology and Metabolic Diseases, Medical University, Lodz, Poland \\ Department of Endocrinology and Metabolic Diseases, Polish Mother's Memorial Hospital Research Institute, Lodz, Poland
}

\begin{abstract}
Sudden cessation of ovary activity as a result of bilateral oophorectomy or chemo- or radiotherapy in premenopausal women is linked with more serious consequences that bear no comparison to natural menopause - to name just a few: higher rate of mortality, higher rate of colorectal and lung cancer, circulatory system diseases, cognitive disorders, Parkinson's disease, psychological disorders, osteoporosis, and sexual disorders. The prolonged period of estrogens deficit in premenopausal age is connected with worsened quality of life.

The progress in oncological care means that in many malignant diseases, also in the case of gynaecological malignancies, the percentage of survivors increases. This makes improving the quality of life more and more important.

The purpose of this review is to establish, based on EBM data, the answer to whether replacement hormonal therapy, being the most effective treatment of menopause symptoms, can be recommended for women who have undergone bilateral oophorectomy because of gynaecological cancer.

On the basis of collected data, derived from meta-analysis, and studies which have been published within the last 20 years, it seems that the use of the appropriate type of hormonal replacement therapy (HRT) in properly selected gynaecological cancer survivors (epithelial ovarian cancer - EOC, endometrial cancer, squamous cell carcinoma of the cervix) is safe and effective.

It seems that benefits connected with better quality of life that stem from the use of appropriate HRT in gynaecological cancer survivors predominate the unfounded fear of disease recurrence in selected patients' groups.
\end{abstract}

Key words: hormonal replacement therapy, menopause, gynaecological cancer survivors.

\section{Introduction}

Menopause can be defined as the permanent cessation of menstruation, connected with reduced activity of the ovaries. Menopause can be due to physiological reasons dependent on age; it can also result from bilateral oophorectomy or be a consequence of postoperative chemo- or radiotherapy in the case of treatment of gynaecological cancers. Cessation of hormonal activity of the ovaries brings with it numerous symptoms and complications that result from drastic reduction of estrogen levels. The reaction of the organism to estrogen deficit remains individual and includes, e.g., hot-flushes, night sweats, mood swings (depression), concentration disturbances, insomnia, vaginal dryness, and/or change in skin mass density (thickness, decreased elasticity) [1]. It can also lead to sexual disorders. Sudden stop of activity of the ovaries, which is the result of bilateral oophorectomy or chemo- or radiotherapy in premenopausal women, is linked with more serious consequences that bear no comparison to natural menopause - to name just a few: higher rate of mortality, higher rate of colorectal and lung cancer, circulatory system diseases, cognitive disorders, Parkinson's disease, psychological disorders, osteoporosis, and sexual disorders [2, 3]. The prolonged period of estrogens deficit in premenopausal age is connected with worsened quality of life [4]. That is why - in order to minimise the effects of lack of estrogens in oncological patients - several European scientific societies, active in the field of gynaecological oncology, radiotherapy, oncology, and pathology, in the period 2014-2018 came up with detailed conditions that must be fulfilled to maintain the function of the ovaries while treating the malignant tumours of reproductive organs and tissues (endometrium, ovaries, cervix), including borderline type ovarian cancer [5-9]. However, premenopausal women who have undergone bilateral oophorectomy because of reproductive organ cancer still suffer from early menopause. Women affected by $B R C A 1 / B R C A 2$ gene mutations, who were subjected to preventive bilateral oophorectomy in reproductive age, face a similar problem. 
The progress in oncological care means that in many tumours - including reproductive organ cancer the percentage of survivors is increasing. This makes improving the quality of life more and more important.

The most effective treatment of estrogen deficit is hormonal replacement therapy (HRT); however, many doctors are reluctant to introduce HRT to gynaecological cancer survivors who have undergone bilateral oophorectomy, because of the risk of recurrence [10]. Moreover, patients receive contradictory information from various specialists concerning treatment [10], which may be a cause of therapy refusal in some of the women subjected to surgical treatment, even though the menopausal symptoms are severe. In the face of divergent results of studies, attempts are being made to develop uniform and generally available guidelines for the use of HRT in this group of women. Despite the variable opinions over a number of years concerning the safety of HRT, the results of studies published today seem to answer more and more questions.

The purpose of this review is to establish, based on EBM data, whether replacement hormonal therapy, being the most effective treatment of menopause symptoms, can be recommended for women who have undergone bilateral oophorectomy because of gynaecological cancer.

\section{Endometrial cancer}

Endometrial cancer is the $6^{\text {th }}$ most common malicious tumour worldwide. It occurs mainly in post-menopausal women; however, about $25 \%$ of cases concern women in premenopausal age and just $5 \%$ at a younger age - before 40 years old [11].

There are 2 types of endometrial cancer: type $1-$ endometrioid adenocarcinoma (EEA), which is the most common (80-90\%) and is hormone-sensitive; and type 2, which comprises serous, clear cell, and undifferentiated carcinomas, as well as carcinoserous/malignant mixed Mullerian tumours [6].

This cancer is considered to be "potentially curable"; hence, more than $75 \%$ of cases are detected at the early stage of development (stage 1). Generally, 5-year survival for all stages of this tumour is $86 \%$, and for stage 1 it increases to $97 \%$ [12].

Although the majority of patients with endometrial type 1 cancer are diagnosed very early with good prognosis, some of them are still failing to reach 5-year overall survival, even those with low-grade or early-stage EEA. This observation may be dependent on estrogen (ER) and progesterone (PR) receptor expression. Currently, ER and PR status have not been implemented into any clinical guidelines; however they seem to be the most validated prognostic biomarkers for endometrial cancer [13].
The main risk factor of endometrial cancer is prolonged exposition to imbalanced estrogens (nulliparity, early menarche, late menopause or prolonged estrogen replacement therapy (ET), diabetes, arterial hypertension, taking medicaments - tamoxifen, selective modulators of Es) [14, 15]. Prolonged combined contraception or estrogen-progestagenic replacement therapy may minimise this risk [16]. The standard procedure in the case of endometrial cancer being diagnosed at an early stage (I or II) is surgical treatment: panhysterectomy. In the case of cancers at middle/high histological risk radio- or brachytherapy is used as a complementary treatment. A consequence of therapy in endometrial cancer, especially in premenopausal women, is the appearance of menopause symptoms; in young women there is a risk of osteoporosis and cardiovascular diseases [17, 18]. Taking into account that most endometrial cancers are estrogen-sensitive, and the fact that prolonged exposition to estrogens increases the risk of disease, the use of ET in women after endometrial cancer potentially causes cancer recurrence as a result of stimulation of cancer cells remaining after treatment $[14,19]$. The ample meta-analyses based on data from research in the years 1986-2019, published in Medline database (PubMed), Web of Science, ClinicalTrial.gov, and Cochrane Library, showed that HRT used in women after endometrial cancer in stage I and II has no negative influence on survival prognosis [20]. The results of some small observational studies indicate that recurrence of cancer is not higher, and the length of life does not change because of the HRT use in that group of women, but in most cases the mentioned parameters undergo improvement in relation to women who did not undergo that treatment [21-27]. It has been shown that complex estrogen-progestagenic therapy has a protective effect in terms of disease recurrence (ER: 0.23; 95\% $\mathrm{Cl}$ : 0.08-0.66), but when introducing only estrogens no such effect was observed (ER: 0.35; 95\%) [27]. The conclusions derived from meta-analysis, based mainly on retrospective studies, suggesting that recurrence and death rates are similar for women who have been treated for early-stage low-risk endometrial cancers (grade 1 and grade 2 endometrioid subtypes with negative ER and PR), if HRT has been applied, led to the softening of the North American Menopause Society position concerning the use of HRT in endometrial cancer survivors. At present, estrogen therapy is not recommended only for women with more advanced stages or higher risk endometrial cancer [28], while - according to the previous therapy position statement from 2012 - estrogen therapy was not recommended in patients treated for all stages of endometrial cancer [29].

Progestogens exert a protective effect by reducing the excessive proliferation of endometrial cells stimulated by estrogens. This process is mediated by a decrease in ER density, inhibition of DNA synthesis 
in endometrial cells, limitation of mitotic activity of ER-controlled transcription oncogenes, and induction of oestradiol degrading enzymes. The effect of progestogen depends on duration of exposure to progestogen. Minimal duration of the therapy is 10 days per month and optimal is 12-14 days per month. Courses of progestogen shorter than 10 days result in proliferation of endometrium in 2-3\% of women annually [30].

Progesterone has been used as the only treatment of menopausal symptoms, and some trials using the transdermal route for longer duration and with oral treatment at higher doses for vasomotor symptoms have shown beneficial effects. Unfortunately, no study has reported an improvement of mood symptoms, and moreover, because of side effects such as headaches and vaginal bleeding, in the majority of studies discontinuation of treatment in 6-21\% of patients was observed [31].

According to the official statement of the European Menopause and Andropause Society, estrogen replacement is a reasonable option for patients who are at low risk of tumour recurrence, but the initiation of such therapy should be individualized and discussed in detail with the patient [32].

\section{Ovarian cancer}

Ovarian cancer is a heterogenic disease. Epithelial ovarian cancer (EOC) can be divided into 5 histologic subtypes: serous tumours, mucinous tumours, endometrioid tumours, clear cell tumours, and other subtypes, which constitutes $90 \%$ of cases, while germ cell tumours and sex cord stromal tumours account for $5 \%$ and $1.2 \%$, respectively [33].

Average age at diagnosis for EOC is about 60 years [34]; however, $40 \%$ of cases concern women in their $30-60$ s, hence $3-17 \%$ of patients - at the moment of diagnosis - are below 40 years of age [34-36]. In most cases, EOC is recognized at an advanced stage (65$70 \%)$. Five-year survival for all stages is $45 \%[35,37]$. The prognosis for EOC in the case of early diagnosis is promising and with low percentages of disease recurrence and mortality (below 10\%) [38].

Aggressive, surgical treatment includes hysterectomy, bilateral oophorectomy, and omentectomy with paraaortic lymph node dissection [39] and results in the sudden occurrence of menopause symptoms, which result from the removal of ovaries, and estrogen deficit [40]. Menopause symptoms are the outcome of surgical treatment and are described as being more intense and long-lasting in comparison to those observed in natural menopause. As a consequence, it is crucial to limit worsening of symptoms, especially in young patients suffering from EOC, in order to enhance the postoperative quality of life.

The uncertainty concerning the use of HRT in women at that age include the potential hormonal stimu- lation of remaining tumour tissue [41, 42], and as well induction of new, hormone-dependent diseases, especially endometrial cancer $[16,43,44]$ or breast cancer $[43,44]$, which is observed in post-menopausal women who undergo protracted oral estrogen therapy [45].

As in vitro tests have shown, exogenous estrogens promote proliferation, "stemness", and invasiveness of $\mathrm{ER}(+)$ tumour cells [46]. In the context of cancer stem cells, "stemness" would refer to the ability of those cancer stem cells.

Despite of the negative influence of HRT on the risk of ovarian cancer in the general population of menopausal women, especially for serous and endometrioid tumours, as demonstrated in a recent meta-analysis [47], many studies concerning postoperative use of HRT in women treated for EOC have reported that HRT application in this group of women does not exert any detrimental effect on the length of survival or the time of disease remission [48-50], and that - on the contrary - it may have a positive influence on the parameters mentioned above [51-53].

It has been shown that the use of HRT does not promote the development and EOC recurrence [54, 55]. However, the time of HRT use in patients with EOC after surgical treatment may substantially influence on the results. Indeed, a strong positive correlation between HRT period and development of ovarian cancer has been documented [56].

Regarding HRT use in the post-operative treatment of germ cell tumours, the higher risk of disease recurrence has not been observed [57]. It may seem that in patients treated with HRT for granuloma, such therapy should not be applied because of the hormonal character of that cancer. There is no direct evidence of a negative, long-lasting hormonal treatment effect on patients' survival; however, the start of HRT in those cases is not considered to be safe $[57,58]$.

The BRCA1 and BRCA2 gene mutations are associated with the increased risk of developing invasive EOC and breast cancer. In patients showing these gene mutations, risk-reducing salpingo-oophorectomy (RRSO) is recommended [59]. However, this leads to the possibility of severe menopausal symptoms; RRSO performed between 30 and 45 years of age seems to be the most effective therapy [59]. Data on HRT after prophylactic oophorectomy are limited, but short-term use of HRT seems to be safe [59]. Moreover, HRT use after RRSO in BRCA1 and BRCA2 mutation carriers does not affect breast cancer risk. Comparison of the different HRT types suggests that estrogens alone should be related to the lowest breast cancer risk $[60,61]$.

\section{Cervical cancer}

The highest rate of cervical cancer cases is noted in the group of women aged 25-29 years, and most cases 
are seen in women before they are 45 years old [33]. Cervical cancer is diagnosed at the early stage in $54 \%$ of women [33], for which the survival rate is $95.9 \%$.

The most common cervical cancer (89-90\%) is the squamous cell carcinoma, which does not show dependency on estrogens. The remaining $10-20 \%$ are cervical adenocarcinomas, which biologically resemble endometrial cancer.

Treatment is dependent on the disease stage and on the FIGO Cancer Report histological type [62].

Patients who have advanced cancer above IB2 stage of squamous cell carcinoma or women with adenocarcinoma in IB stage and frequent metastasis, which is characteristic for that type of tumour [37], are prone to ovary damage during complementary radio- or chemotherapy or after ovary removal.

Squamous cell carcinoma is not considered to be a hormone-dependent tumour, although the presence of $E R$ and $P R$ receptors was indicated in the uterine cervix [63]. Moreover, the long-term use of oral contraception increases the risk of cervical cancer [64].

Available studies do not confirm the negative influence of systemic HRT use on patients' survival and suffering from the squamous cell carcinoma, and the disease recurrence is absent [65].

The local use of estrogens turned out to effectively reduce the frequency of urogenital complications, which happened to be a consequence of vaginal atrophy after radiotherapy [65]. Complications after radiation were lighter and lasted less time in comparison to women from a control group who did not undergo therapy. Studies have shown that after 2 weeks of local use of oestradiol, its concentration in serum decreased to the level which is normally observed in postmenopausal women. It was connected to the restricted absorption of medicaments into general circulation, which was the result of vaginal atrophy $[66,67]$. There is no evidence that the local use of estrogens has a detrimental effect on the course of cervical cancer [37].

What is more, it has been shown that the use of complex ER-PR HRT increases the survival and lowers the percentage of recurrence of both types of histological tumours [65].

In the case of adenocarcinoma, positive but statistically invariable correlation between tumour occurrences and the exclusive use of estrogen HRT was observed. In patients with squamous cell carcinoma, such a correlation is absent [68].

In women being treated for squamous cell carcinoma, the use of HRT seems to be safe, although studies concerning the role of estrogens in the pathogenesis of adenocarcinoma, and insufficient number of reports concerning safety of HRT use in women being treated for cervical adenocarcinoma, indicate that considerable caution must be paid concerning treatment selection.
An interesting alternative for cervical cancer survivors, especially survivors of adenocarcinomas, is administration of tibolone - a synthetic steroid with estrogenic, androgenic, and progestogenic characteristics. The use of that drug does not affect survival, but effectively relieves symptoms of surgical menopause [69].

\section{Conclusions}

On the basis of collected data, derived from meta-analysis, and studies which have been published within the last 20 years, it seems that the use of the appropriate type of HRT in properly selected gynaecological cancer survivors (EOC, endometrial cancer, squamous cell carcinoma of the cervix) may be safe and effective. However, the management of menopausal symptoms in gynaecological cancer survivors depends on their age, tumour type, and stage and should be individualized [32]. Attention should be paid to potential limitations that are to be considered while interpreting obtained results, e.g. limited test groups, too little observation time, various times of HRT use, different forms of HRT [70], individual cancer stage, accompanying diseases [71], and heterogeneous ages of patients [51]. Nevertheless, progress in diagnostics that enables early recognition of malignancies and better treatment outcome is crucial to assure a better quality of life to patients with premature surgically induced menopause and subsequent health problems. The benefits connected with better quality of life, which stem from the use of appropriate HRT in gynaecological cancer survivors, outweigh the unjustified fear of disease relapse in selected patient groups. However, the decision to use HRT should be adapted to the individual patient. It should be considered that all survivors of cancer are not the same. Some of them are exhausted from their menopausal symptoms and are willing to accept any amount of risk, while others may have minimal symptoms or may not be willing to accept even a theoretically increased risk of recurrent cancer. Non-hormonal treatment should always be considered as an alternative therapy [72]. Moreover, because the bulk of evidence suggests that type, dose, route, and time of initiation of HRT may greatly affect the risk-benefit profile, additional research is needed to understand how these factors may impact the treatment of women with a history of gynaecological cancer, for whom HRT may play an instrumental role in improving their quality of life.

\section{Acknowledgment}

Preparation of this review was supported by statutory funds from the Polish Mother's Memorial Hospital - Research Institute. 


\section{Disclosure}

The authors report no conflict of interest.

\section{References}

1. Santoro N, Epperson CN, Mathews SB. Menopausal symptoms and their management. Endocrinol Metab Clin N Am 2015; 44: 497-515.

2. Rosner B, Colditz GA. Age at menopause: imputing age at menopause for women with a hysterectomy with application to risk of postmenopausal breast cancer. Ann Epidemiol 2011; 21: 450-460.

3. Erekson EA, Martin DK, Ratner ES. Oophorectomy: the debate between ovarian conservation and elective oophorectomy. Menopause 2013; 20: 110-114.

4. Ossewaarde ME, Bots MI, Verbeek Al, et al. Age at menopause, causespecific mortality and total life expectancy. Epidemiology 2005; 16: 556562.

5. Cibula D, Pötter R, Planchamp F, et al. The European Society of Gynaecological Oncology/European Society for Radiotherapy and Oncology/European Society of Pathology guidelines for the management of patients with cervical cancer. Virchows Arch 2018; 472: 919-936.

6. Colombo N, Creutzberg C, Amant F, et al. ESMO-ESGO-ESTRO Consensus Conference on Endometrial Cancer: diagnosis, treatment and follow-up. Ann Oncol 2016; 27: 16-41.

7. Baert T, Essen-Mitte K, Banerjee S, et al. ESMO-ESGO Consensus Conference Recommendations on Ovarian Cancer: pathology and molecular biology, early and advanced stages, borderline tumours and recurrent disease. Ann Oncol 2019; 30: 672-705.

8. Skirnisdottir I, Garmo H, Wilander E, et al. Borderline ovarian tumors in Sweden 1960-2005: Trends in incidence and age at diagnosis compared to ovarian cancer. Int J Cancer 2008; 123: 1897-1901.

9. Harter P, Gershenson D, Lhommé C, et al. Gynecologic Cancer InterGroup (GCIG) consensus review for ovarian tumors of low malignant potential (borderline ovarian tumors). Int J Gynecol Cancer 2014; 24: 5-8.

10. Halldorsdottir S, Dahlstrand H, Stalberg K. Gynecologists are afraid of prescribing hormone replacement to endometrial/ovarian cancer survivors despite national guidelines a survey in Sweden. Ups J Med Sci 2018; 123: 225-229.

11. Pecorelli S, Pasinetti B, Angioli R, et al. Systemic therapy for gynecologi cal neoplasms: ovary, cervix and endometrium. Cancer Chemotherapy Biol Res Modifiers 2005; 22: 515-544.

12. Sonoda Y, Barakat R. Screening and the prevention of gynecologic cancer: endometrial cancer. Best Pract Res Clin Obstet Gynaecol 2006; 20: 363-377.

13. Guan J, Xie I, Luo X, et al. The prognostic significance of estrogen and progesterone receptors in grade I and II endometrioid endometrial adenocarcinoma: hormone receptors in risk stratification. J Gynecol Oncol 2019; 30: e13

14. Akhmedkhanov A, Zeleniuch-Jacquotte A, Toniolo P. Role of exogenous and endogenous hormones in endometrial cancer. Review of the evidence and research perspectives. Ann New York Acad Sci 2001; 943 : 296-315

15. Siegel RL, Miller KD, Jemal A. Cancer statistics 2015. Cancer J Clin 2015; 65: 5-29.

16. Chlebowski RT, Anderson GL, Sarto GE, et al. Continuous combined es trogen plus progestin and endometrial cancer: the Women's Health Initiative Randomized Trial. J National Cancer Institute 2015; 108: djv350.

17. Muka T, Oliver-Williams C, Kunutsor S, et al. Association of age at onset of menopause and time since onset of menopause with cardiovascular outcomes, intermediate vascular traits, and all-cause mortality: a systematic review and meta-analysis. JAMA Cardiology 2016; 1: 767-776.

18. Svejme O, Ahlborg HG, Nilsson JÅ, et al. Early menopause and risk of osteoporosis, fracture and mortality: a 34-year prospective observational study in 390 women. Br J Obstet Gyneacol 2012; 119: 810-816.

19. Shuster LT, Rhodes DJ, Gostout BS, et al. Premature menopause or early menopause: long-term health consequences. Maturitas 2010; 65: 161166.

20. Di Donato V, Palaia I, D'Aniello D, et al. Does hormone replacement therapy impact the prognosis in endometrial cancer survivors? Oncology 2020; 98 : 195-201.
21. Creasman WT, Henderson D, Hinshaw W. Estrogen replacement therapy in the patient treated for endometrial cancer. Obstet Gynecol 1986; 67: 326-330.

22. Lee RB, Burke TW, Park RC. Estrogen replacement therapy following treatment for stage I endometrial carcinoma. Gynecol Oncol 1990; 36: 189-191.

23. Chapman JA, DiSaia PJ, Osann K, et al. Estrogen replacement in surgical stage I and II endometrial cancer survivors. Am J Obstet Gynecol 1996; 175: 1195-1200.

24. Suriano KA, McHale M, McLaren CE, et al. Estrogen replacement therapy in endometrial cancer patients: a matched control study. Obstet Gynecol 2001; 97: 555-560.

25. Ayhan A, Taskiran C, Simsek S, et al. Does immediate hormone replacement therapy affect the oncologic outcome in endometrial cancer survivors? Int J Gynecol Cancer 2006; 16: 805-808.

26. Barakat RR, Bundy BN, Spirtos NM, et al. Gynecologic Oncology Group Study. Randomized double-blind trial of estrogen replacement therapy versus placebo in stage I or II endometrial cancer: a Gynecologic Oncology Group Study. J Clin Oncol 2006; 24: 587-592.

27. Shim SH, Lee SJ, Kim SN. Effects of hormone replacement therapy on the rate of recurrence in endometrial cancer survivors: a meta-analysis. Eur J Cancer 2014; 50: 1628-1637.

28. Position Statement. The 2017 hormone therapy position statement of The North American Menopause Society. Menopause 2017; 24: 728-753.

29. Position Statement. The 2012 hormone therapy position statement of The North American Menopause Society. Menopause 2012 ;19: 257-271.

30. Witczak K, Sajdak S, Kojs Z. Hormonalna terapia zastępcza w ginekologii onkologicznej. Curr Gynecol Oncol 2013; 11: 62-73.

31. Dolisky SN, Christina CM, Sheehan SS, et al. Efficacy of progestin-only treatment for the management of menopausal symptoms: a systematic review. Menopause 2020; 28: 217-224.

32. Reesa M, Angiolib R, Colemanc R, et al. European Menopause and Andropause Society (EMAS) and International Gynecologic Cancer Society (IGCS) position statement on managing the menopause after gynecological cancer: focus on menopausal symptoms and osteoporosis. Maturitas 2020; 134: 56-61.

33. Richardson A, Ayres J, Cust M, et al. Hormone replacement therapy following treatment of gynecological malignancies. Obstet Gynecol 2019; 21: 291-298.

34. Michaelson-Cohen R, Beller U. Managing menopausal symptoms after gynaecological cancer. Curr Opin Oncol 2009; 21: 407-411.

35. Ibeanu O, Modesitt SC, Ducie J, et al. Hormone replacement therapy in gynecologic cancer survivors: why not? Gynecol Oncol 2011; 122: $447-$ 454

36. Wen $\mathrm{Y}$, Huang $\mathrm{H}, \mathrm{Wu} \mathrm{M}$, et al. The safety of postoperative hormone replacement therapy in epithelial ovarian cancer patients in China. Climacteric 2013; 16: 673-681.

37. Singh P, Oehler MK. Hormone replacement after gynaecological cancer. Maturitas 2010; 65: 190-197.

38. Lowe KA, Chia VM, Taylor A, et al. An international assessment of ovarian cancer incidence and mortality. Gynecol Oncol 2013; 130: 107-114.

39. Fleming G, Seidman J, Lengyel E. Epithelial ovarian cancer. In: Barakat RR, Berchuck A, Markman M, Randall ME (eds.). Principles and Practice of Gynecologic Oncology. 6th ed. Philadelphia (PA): Lippincott Williams and Wilkins 2013: 757-847.

40. van Der Vort DJ, van Der Weijer PH, Barentsen R. Early menopause: increased fracture risk at older age. Osteoporos Int 2003; 14: 525-530.

41. Braem MG, Onland-Moret NC, van den Brandt PA, et al. Reproductive and hormonal factors in association with ovarian cancer in the Netherlands cohort study. Am J Epidemiol 2010; 172: 1181-1189.

42. Zhou B, Sun Q, Cong R, et al. Hormone replacement therapy and ovarian cancer risk: a meta-analysis. Gynecol Oncol 2008; 108: 641-651.

43. Benkhadra K, Mohammed K, Nofal AA, et al. Menopausal hormone therapy and mortality: a systematic review and meta-analysis. J Clin Endocrin Metab 2015; 100: 4021-4028.

44. Manson JE, Chlebowski RT, Stefanick ML, et al. Menopausal hormone therapy and health outcomes during the intervention and extended poststopping phases of the Women's Health Initiative randomized trials. J Am Med Assoc 2013; 310: 1353-1368.

45. Hinds L, Price J. Menopause, hormone replacement and gynaecological cancers. Menopause Int 2010; 16: 89-93. 
46. Sun Y, Wang C, Fan P, et al. Estrogen promotes stemness and invasiveness of ER - positive breast cancer cells through Gli1 activation. Mol Cancer 2014; 13: 137-152.

47. Liu Y, Ma L, Yang X, et al. Menopausal hormone replacement therapy and the risk of ovarian cancer: a meta-analysis. Front Endocrinol (Lausanne) 2019; 10: 801

48. Li L, Pan Z, Gao K, et al. Impact of postoperative hormone replacement therapy on life quality and prognosis in patients with ovarian malig nancy. Oncol Lett 2012; 3: 244-249.

49. Hopkins ML, Fung MF, Le T, et al. Ovarian cancer patients and hormone replacement therapy: a systematic review. Gynecol Oncol 2004; 92: 827-832.

50. Levgur M. Estrogen and combined hormone therapy for women after genital malignancies. J Reprod Med 2004; 49: 837-848.

51. Li D, Ding C-Y, Qiu L-H. Postoperative hormone replacement therapy for epithelial ovarian cancer patients: a systematic review and meta-analysis. Gynecol Oncol 2015; 139: 355-362.

52. Mascarenhas C, Lambe M, Bellocco R, et al. Use of hormonal replacement therapy before and after ovarian cancer diagnosis and ovarian cancer survival. Int J Cancer 2006; 119: 2907-2015.

53. Eeles RA, Morden JP, Gore M, et al. Adjuvant hormone therapy may improve survival in epithelial ovarian cancer: results of the AHT randomised trial. J Clin Oncol 2015; 33: 4138-4144.

54. Bebar S, Ursic-Vrscaj M. Hormone replacement therapy after epithelial ovarian cancer treatment. Eur J Gynaecol Oncol 2000; 21: 192-196.

55. Ursic-Vrscaj M, Bebar S, Zakelj MP. Hormone replacement therapy after invasive ovarian serous cystadenocarcinoma treatment: the effect on survival. Menopause 2001; 8: 70-75.

56. Lacey JV, Mink PJ, Lubin JH, et al. Menopausal hormone replacement therapy and risk of ovarian cancer. JAMA 2002; 288: 334-341.

57. Kuhle CL, Kapoor E, Sood R, et al. Menopausal hormone therapy in cancer survivors: a narrative review of the literature. Maturitas 2016; 92 86-96

58. Del Carmen MG, Rice LW. Management of menopausal symptoms in women with gynecologic cancers. Gynecol Oncol 2017; 146: 427-435.

59. Vermeulen RFM, Korse CM, Kenter GG, et al. Safety of hormone replacement therapy following risk-reducing salpingo-oophorectomy: systematic review of literature and guidelines. Climacteric 2019; 22: 352-360.

60. Marchetti C, De Felice F, Boccia S, et al. Hormone replacement therapy after prophylactic risk-reducing salpingo-oophorectomy and breast cancer risk in $B R C A 1$ and $B R C A 2$ mutation carriers: a meta-analysis. Crit Rev Oncol Hematol 2018; 132: 111-115.

61. Kotsopoulos J, Gronwald J, Karlan BY, et al. Hormone replacement therapy after oophorectomy and breast cancer risk among BRCA1 mutation carriers. JAMA Oncol 2018; 4: 1059-1065.

62. FIGO Cancer Report 2018. Int J Gynecol Obstet 2018; 143: 1-158.

63. Bodner K, Laubichler P, Kimberger O, et al. Oestrogen and progesterone receptor expression in patients with adenocarcinoma of the uterine cervix and correlation with various clinicopathological parameters. Anticancer Res 2010; 30: 1341-1345.

64. Smith JS, Green J, Berrington de Gonzales A, et al. Cervical cancer and use of hormonal contraceptives: a systematic review. Lancet 2003; 361: 1159-1167.

65. Ploch E. Hormonal replacement therapy in patients after cervical cancer treatment. Gynecol Oncol 1987; 26: 169-177.

66. Suckling J, Lethaby A, Kennedy R. Local estrogen for vaginal atrophy in postmenopausal women. Cochrane Database Syst Rev 2006: CD001500.

67. Nilsson K, Heimer G. Low-dose 17 beta-oestradiol during maintenance therapy - a pharmacokinetic and pharmacodynamic study. Maturitas 1995; 21: 33-38.

68. Lacey JV Jr, Brinton LA, Barnes WA, et al. Use of hormone replacement therapy and adenocarcinomas and squamous cell carcinomas of the uterine cervix. Gynecol Oncol 2000; 77: 149-154.

69. Lee S-H, Cho Y-J, Cho K-J, et al. Effect of tibolone on the survival of early stage cervical adenocarcinoma patients. Obstet Gynecol Sci 2018; 61: 584-589.

70. Saeaib N, Peeyananjarassri K, Liabsuetrakul T, et al. Hormone replacement therapy after surgery for epithelial ovarian cancer (Protocol) Cochrane Database of Systematic Reviews 2017, Issue 2. Art. No: CD012559.
71. Deli T, Orosz M, Jakab A. Hormone replacement therapy in cancer survivors - review of the literature. Path Oncol Res 2020; 26: 63-78.

72. Nonhormonal management of menopause-associated vasomotor symptoms. 2015 position statement of The North American Menopause Society. Menopause 2015; 22: 1155-1172. 